\title{
Record linkage study of hypokalaemia in hospitalized patients
}

\author{
B.J. Paice, K.R. Paterson, F. Onyanga-Omara, T. Donnelly, J.M.B. Gray and \\ D.H. Lawson
}

Royal Infirmary, Glasgow G4 OSF, Scotland, UK

\begin{abstract}
Summary: Retrospective analysis of biochemical data from 58,167 hospital inpatients revealed that $21 \%$ developed hypokalaemia during hospitalization - in $5.2 \%$ the serum potassium was less than $3.0 \mathrm{mmol} / \mathrm{l}$. Subsequent evaluation showed a positive correlation between hypokalaemia and both female sex and hospital mortality. Patients with leukaemia and lymphoid tumours, especially when receiving antibiotic or cytotoxic therapy, and patients with gastro-intestinal malignancy were amongst those most frequently experiencing hypokalaemia. There was no significant association with cardiovascular disease. Drug and intravenous fluid administration accounted for the hypokalaemia in $56 \%$ of patients. While drug-related hypokalaemia was most commonly seen with diuretics, it was also apparent following use of steroids, insulin and haematinics.
\end{abstract}

\section{Introduction}

Glasgow Royal Infirmary is a large teaching hospital serving a predominantly urban population. We have reviewed details of all patients in a 3 year period who had at least one estimation of serum electrolyte concentrations. From this cohort we have previously reported details of 73 patients who had at least one recorded value of serum potassium concentration below $2.0 \mathrm{mmol} / 1$ (Lawson et al., 1979). This profound hypokalaemia was most frequently observed in female patients and the frequency of acute myeloid leukaemia was greater than in a normokalaemic control population. In contrast, the frequency of a primary diagnosis of cardiovascular disease was not increased in patients with profound hypokalaemia.

The present report describes further analysis of the initial cohort of patients with particular attention to subjects with lesser degrees of hypokalaemia.

\section{Subjects, methods and analysis}

The methods of this study have been described in detail previously (Lawson et al., 1979). Briefly, the Biochemistry Department of Glasgow Royal Infirmary maintains a computerized record system which includes details of each patient's sex, ward and electrolyte concentrations by date and links this data

Correspondence: K.R. Paterson, M.B., M.R.C.P.

Accepted 25th September, 1985 to the patient's individual hospital number. Details of all patients discharged from Scottish hospitals are computerized for central analysis by the Scottish Home \& Health Department (Heasman, 1968). Information on each patient's age, sex, status on discharge, duration of admission and discharge diagnosis is recorded, in addition to details of any operative procedures undertaken during the admission. This record also includes the patient's hospital number. Thus using the patients' hospital numbers as identification, it was possible to combine information from both computers for detailed analyses.

During the 3 year period studied, 58,167 patients had at least one estimation of serum electrolytes entered into the biochemistry computer record. Examining these results shows that the lowest serum potassium $\left(\mathrm{K}^{+}-\right.$laboratory normal reference range $3.5-5.0 \mathrm{mmol} / \mathrm{l}$ ) was less than $2.0 \mathrm{mmol} / 1$ in 73 patients (Set A), $2.0-2.4 \mathrm{mmol} / 1$ in 472 (Set B), 2.5-2.9 mmol/1 in 2,498 (Set C) and 3.0-3.4 mmol/1 in 9,345 (Set $D$ ), the sets being mutually exclusive.

Because of the large numbers involved, a random sample group of patients was selected from each of sets B, C and D; a similar random sample of normokalaemic patients was also selected giving five groups for further study. Group A: all 73 patients with at least one recorded value of serum potassium concentration below $2.0 \mathrm{mmol} / 1$; Group B: 219 patients selected randomly from the 472 who had a lowest recorded value of $\mathrm{K}^{+}$between 2.0 and $2.4 \mathrm{mmol} / 1$; Group C: 219 
Table I Characteristics of five sample groups of patients studied

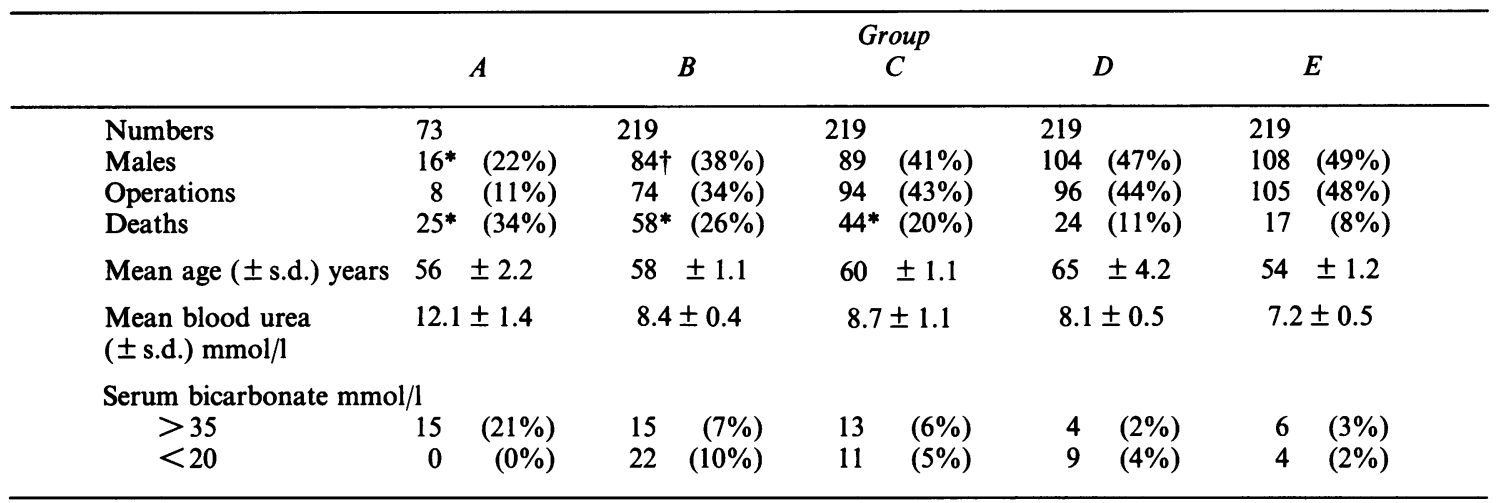

* $-P<0.001 ; \uparrow P<0.025$ compared with normokalaemic subjects (Group E).

patients selected from the 2,498 who had a lowest recorded value of $\mathrm{K}^{+}$between 2.5 and $2.9 \mathrm{mmol} / \mathrm{l}$; Group D: 219 patients selected from the 9,345 who had a lowest recorded value of $\mathrm{K}^{+}$between 3.0 and $3.4 \mathrm{mmol} / \mathrm{l}$; Group E: 219 patients selected from the remaining group whose serum plasma potassium concentration was $3.5 \mathrm{mmol} / 1$ or greater. In each of the Groups B-E 219 patients were selected to provide a ratio of 3:1 with those in Group A.

Details of age, sex, status on discharge, primary discharge diagnosis and surgical operations during admission were obtained for each member of the five groups. The serum urea and bicarbonate concentrations at the time of the serum potassium estimation were also noted.

More detailed investigation (from scrutiny of individual case records) of the hypokalaemia in 64 of the 73 patients in Group A has been reported previously (Lawson et al., 1979). For the present investigation, a random sample of 64 patients was obtained separately from each of groups B, C and D for similar detailed assessment. Since only $80 \%$ of records sought were available - the others being misplaced for a variety of administrative reasons - this random sample involved a degree of selection on the basis of availability of case notes. However, scrutiny of the basic computerized information on patients whose records were sought but not obtained, revealed no evidence of bias with respect to age, sex, hospital ward or discharge diagnosis.

Results were analysed with a $\chi^{2}$ test applied across the five groups of patients studied (degrees of freedom $=4$ ). In addition, individual $\chi^{2}$ test werec applied comparing groups $\mathrm{A}, \mathrm{B}, \mathrm{C}$ and $\mathrm{D}$ individually ? with the group of normokalaemic subjects - results are expressed as two-tailed ' $P$ ' values.

\section{Results}

The distribution of patients in Groups $\mathrm{A}-\mathrm{E}$ with regard to age, sex, mortality and serum urea and bicarbonate concentrations on day of lowest recorded serum potassium concentration are shown in Table I. There were strong positive associations between hypokalaemia and both female sex $\left(x^{2}=20.9\right.$, df $=4$,

Table II Distribution of patients with hypokalaemia with respect to primary discharge diagnosis (based on ICD Classification)

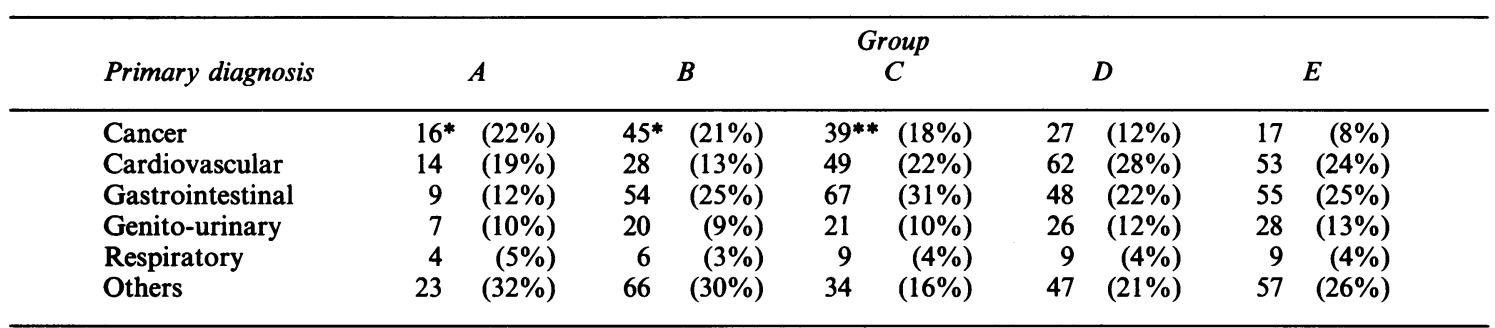

$* P<0.001 ;{ }^{* *} P<0.005$ compared with normokalaemic subjects (Group E). 
Table III Relationship between primary discharge diagnosis of cancer and hypokalaemia

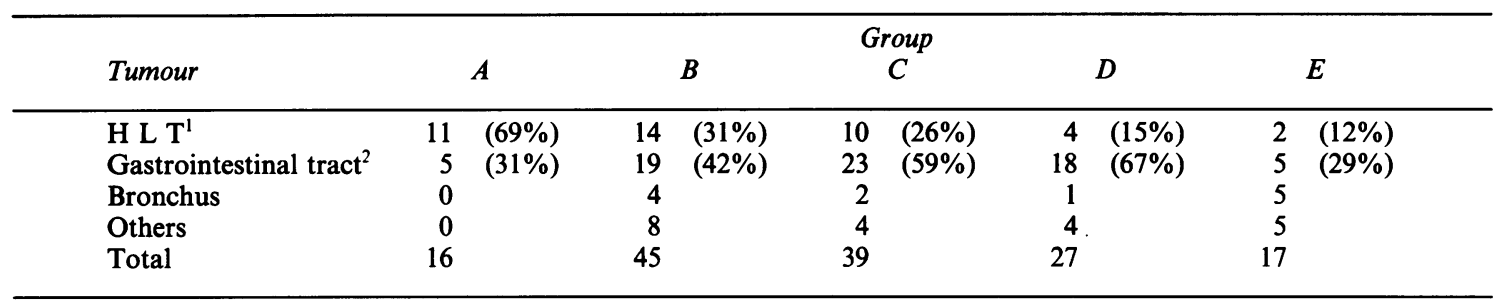

${ }^{1}$ Tumours of haemopoietic and lymphatic tissues.

${ }^{2}$ Includes stomach, colon and rectum.

$P<0.001)$ and death during hospitalization $\left(\chi^{2}=47.9\right.$, df $\left.=4, P<0.0005\right)$. A strong negative relationship between hypokalaemia and surgical operations was also demonstrated. The relationship between age and degree of hypokalaemia was complex, reflecting the varying causes underlying the development of the hypokalaemia. Significant disturbance of acid-base balance was demonstrated only in Group A, $21 \%$ of patients having an elevated serum bicarbonate.

The distribution of primary discharge diagnoses is shown in Table II. There was a strong and consistent association between hypokalaemia and a primary discharge diagnosis of cancer $\left(\chi^{2}=19.4, \mathrm{df}=4\right.$, $P<0.001)$. Table III shows the nature of the cancer in the 144 patients with malignant disease. It is clear that haematological malignancies account for most of the profound hypokalaemia (Group A) while neoplasms of the gastrointestinal tract are more common in patients with lesser degrees of hypokalaemia (Groups $\mathrm{B}, \mathrm{C}$ and D). There was no obvious relationship with any other type of tumour. The distribution of other primary diagnoses was not significantly related to the degree of hypokalaemia (Table II) - of particular interest was the fact that only in Group D was the frequency of cardiovascular disease greater than in the normokalaemic group, a marginal excess which could easily have arisen by chance.

Detailed review of case records of a sample of 64 patients in each of the four Groups A-D indicated the likely cause of the hypokalaemia in the majority of patients $(243 / 256=95 \%)$ (Table IV).

Table IV Apparent cause of hypokalaemia in relation to degree of hypokalaemia (64 patients in each group)

\begin{tabular}{|c|c|c|c|c|c|c|c|c|}
\hline \multirow{2}{*}{$\begin{array}{l}\text { Causes of hypokalaemia } \\
\text { Diuretics }\end{array}$} & \multicolumn{2}{|c|}{$<2.0 \mathrm{mmol} / \mathrm{l}$} & \multicolumn{2}{|c|}{$2.0-2.4 \mathrm{mmol} / \mathrm{l}$} & \multicolumn{2}{|c|}{$2.5-2.9 \mathrm{mmol} / \mathrm{l}$} & \multicolumn{2}{|c|}{$3.0-3.4 \mathrm{mmol} / \mathrm{l}$} \\
\hline & 10 & $(16 \%)$ & 14 & $(22 \%)$ & 19 & $(29 \%)$ & 18 & $(28 \%)$ \\
\hline Steroids & 1 & $(1 \%)$ & 4 & $(6 \%)$ & 2 & $(3 \%)$ & 2 & $(3 \%)$ \\
\hline Insulin & 3 & $(5 \%)$ & 1 & $(1 \%)$ & 1 & $(1 \%)$ & 2 & ( $3 \%)$ \\
\hline Peptic ulcer therapy & 3 & $(5 \%)$ & 0 & - & 0 & - & 0 & - \\
\hline Clindamycin & 2 & $(3 \%)$ & 0 & - & 3 & $(5 \%)$ & 0 & - \\
\hline $\begin{array}{l}\text { Hydroxocobalamin } \\
\pm \text { folic acid }\end{array}$ & 0 & - & 3 & $(5 \%)$ & 3 & $(5 \%)$ & 1 & $(1 \%)$ \\
\hline Laxatives & 0 & - & 2 & $(3 \%)$ & 1 & $(1 \%)$ & 1 & $(1 \%)$ \\
\hline Others & 2 & $(3 \%)$ & 0 & - & 0 & - & 0 & - \\
\hline Drug therapy & 21 & $(33 \%)$ & 24 & $(37 \%)$ & 29 & $(45 \%)$ & 24 & $(37 \%)$ \\
\hline $\begin{array}{l}\text { IV fluids with insufficient } \\
\text { potassium replacement }\end{array}$ & 11 & $(17 \%)$ & 10 & $(16 \%)$ & 14 & $(22 \%)$ & 11 & $(17 \%)$ \\
\hline Gastrointestinal losses & 9 & $(14 \%)$ & 16 & $(26 \%)$ & 11 & $(17 \%)$ & 7 & $(11 \%)$ \\
\hline Acute leukaemias & 8 & $(12 \%)$ & 0 & - & 0 & - & 0 & - \\
\hline Inadequate intake & 6 & $(9 \%)$ & 5 & $(8 \%)$ & 6 & $(9 \%)$ & 7 & $(11 \%)$ \\
\hline Liver disease & 4 & $(6 \%)$ & 5 & $(8 \%)$ & 2 & $(3 \%)$ & 3 & $(5 \%)$ \\
\hline Urinary $\mathrm{K}^{+}$losses & 4 & $(6 \%)$ & 1 & $(1 \%)$ & 0 & - & 1 & $(1 \%)$ \\
\hline Burns & 1 & $(1 \%)$ & 0 & - & 1 & $(1 \%)$ & 2 & $(3 \%)$ \\
\hline Cause indeterminate & 0 & - & 3 & $(5 \%)$ & 1 & $(1 \%)$ & 9 & $(14 \%)$ \\
\hline
\end{tabular}


Overall, drug therapy was implicated in $38 \%$ of patients. Of the drugs incriminated in causing hypokalaemia, diuretics accounted for $62 \%$, steroids $9 \%$ and insulin and hydroxocobalamin 7\% each.

Diuretics were the likely cause of hypokalaemia in 61 patients. There was a tendency for these patients to have mild-moderate $(2.5-3.4 \mathrm{mmol} / 1-37$ patients $)$ rather than severe hypokalaemia $(<2.4 \mathrm{mmol} / \mathrm{l}-24$ patients) $\left(\chi^{2}=3.64, P<0.06\right.$ comparing groups $A+B$ with groups $\mathbf{C}+D)$. There was no clear trend towards any particular group of diuretics causing hypokalaemia, both thiazides and loop diuretics being approximately equally represented.

Corticosteroid (hydrocortisone or prednisolone) and corticotrophin use accounted for nine patients with hypokalaemia. There was no relationship between the magnitude of the hypokalaemia and the drug or dosage of drug administered. In this series, steroidinduced hypokalaemia arose almost exclusively in patients with haematological malignancies.

Soluble insulin given during treatment of diabetic ketoacidosis was associated with hypokalaemia in seven subjects, in three of whom profound hypokalaemia was recorded. One of the latter three subjects was a patient who received insulin to correct diazoxide-induced hyperglycaemia, thereby experiencing two life-threatening drug-related events during the course of a single hospital admission.

Seven patients developed hypokalaemia during initial therapy for megaloblastic anaemia. In four of these patients, the presenting haemoglobin level was below $8 \mathrm{~g} / \mathrm{dl}$ and in the other three, thrombocytopaenia (platelets $<50 \times 10^{9} / 1$ ) and or leucopaenia $\left(\mathrm{WBC}<1.5 \times 10^{9} / 1\right)$ was a feature of the presentation. Two of these patients developed megaloblastic anaemia secondary to long-term treatment with anticonvulsant drugs.

In addition to hypokalaemia arising as a direct result of drug treatment, 46 patients developed hypokalaemia of varying severity while receiving intravenous fluids with insufficient potassium replacement many of these patients had underlying malignant disease. If these patients are included in the group with drug-related hypokalaemias, they bring the total to 144 of the 256 patients $(56 \%)$.

\section{Discussion}

In this three year study of hypokalaemia in a large Scottish teaching hospital, $21 \%$ of patients were noted to have a serum potassium concentration of less than $3.5 \mathrm{mmol} / 1$ during their admission - in $5.2 \%$ of the study cohort the hypokalaemia was potentially clinically significant (serum potassium $<3.0 \mathrm{mmol} / \mathrm{l}$ ). Hypokalaemia was associated with a significantly increased hospital mortality. It was also commoner in females - this finding is unlikely to have arisen by chance in view of the strength of the statistical relationship and was not the result of confounding due to age or diagnosis. This association has also been reported from Denmark (Krakauer \& Lauritzen, 1978) and appears to be real. Its aetiology is unclear, but it is possible that undeclared laxative abuse and hormonal influences may play a role.

Hypokalaemia has been reported in acute myeloid leukaemia (Kassirer \& Harrington, 1977; Pickering \& Catovsky, 1973; Ledoux et al., 1978). This occurs partly as a consequence of chronic lysozymuria resulting in renal tubular damage and subsequent excessive renal potassium elimination, and partly as a consequence of concurrent treatment with drugs such as cytotoxic agents (Schreiner \& Maher, 1965) and some antibiotics (Young et al., 1973; Tattersall et al., 1970). In the present study, severe hypokalaemia has been noted in patients receiving cytotoxic treatment for several types of acute leukaemia and lymphoid tumours, this sub-group comprising one of the largest single groups of patients in the study. Particular care and attention to potassium status is thus required for all patients receiving cytotoxic or antibiotic treatment for haemopoietic or lymphoid tumours.

Hypokalaemia was also noted in patients with gastrointestinal tumours but this was of lesser severity and presumably reflects loss of potassium from the gut.

Of particular interest in view of the known propensity of diuretic drugs to cause mild hypokalaemia (Healy et al., 1970; Down et al., 1972) was the demonstration of only a small and statistically insignificant excess of primary cardiovascular disease in the patients with hypokalaemia. However, on reviewing the apparent causes of hypokalaemia in a random sample of 256 patients there were $61(24 \%)$ in whom diuretics, both thiazides and loop diuretics, were incriminated as the cause of hypokalaemia. In most subjects the hypokalaemia was slight, thus confirming the views of Morgan \& Davidson (1980) in Leeds and Kassirer \& Harrington (1977) in Boston.

Hypokalaemia is a well-recognized consequence of correction of ketoacidosis in diabetes mellitus (Nabarro et al., 1952) and of response to haematinics in megaloblastic anaemia (Lawson et al., 1972). Despite this, some patients continue to develop moderate or severe hypokalaemia under these circumstances. Such hypokalaemia is eminently avoidable and consideration should be given to routine use of potassium supplementation in such patients.

A similar consideration applies to hypokalaemia developing during intravenous fluid therapy. The widespread availability of prepared packs of intravenous fluid containing potassium has greatly facilitated potassium administration in intravenous fluid regimens and it should be routine policy to ensure a daily 
potassium input of at least $40 \mathrm{mmol}$ unless a specific contraindication such as pre-existing hyperkalaemia or acute renal failure exists. Close monitoring of electrolyte concentrations during intravenous fluid therapy is mandatory and special attention to potassium status is required in view of the risk of both hypoand hyperkalaemia.

Record linkage has proved useful in analysing the frequency and aetiology of hypokalaemia. We conclude that minor degrees of hypokalaemia are com-

\section{References}

DOWN, P.E., POLAK, A., RAD, R. \& MEAD, J.A. (1972). Fate of potassium supplements in six patients receiving long-term diuretics for oedematous disease. Lancet, ii, 721.

HEALY, J.J., McKENNA, T.J., CANNING, B.S.E.J., BRIEN T.G., DUFFY, G.J. \& MULDOWNEY, F.P. (1970). Body composition changes in hypertensive subjects on long-term oral diuretic therapy. British Medical Journal, 1, 716.

HEASMAN, M.A. (1968). Scottish hospital in-patient statistics - sources and uses. Health Bulletin, 26, 10.

KASSIRER, J.P. \& HARRINGTON, J.T. (1977). Diuretics and potassium metabolism: a reassessment of the need, effectiveness and safety of potassium therapy. Kidney International, 11, 505.

KRAKAURER, R. \& LAURITZEN, M. (1978). Diuretic therapy and hypokalaemia in geriatric outpatients. Danish Medical Bulletin, 25, 26.

LAWSON, D.H., MURRAY, R.M. \& PARKER, J.L.W. (1972). Early mortality in the megaloblastic anaemias. Quarterly Journal of Medicine, 41, 1.

LAWSON, D.H., HENRY, D.A. \& LOWE, J.M. (1979). Severe hypokalaemia in hospitalised patients. Archives of Internal Medicine, 139, 978.

LEDOUX, F., BERGERAT, J.-P., VETTER, J.-M., LANG, J.-M. \& mon in hospitalized patients and that over $5 \%$ of patients develop potentially clinically significant hypokalaemia. Hypokalaemia is commoner in female patients and in patients with malignant disease and mortality is significantly increased in patients with $\mathrm{K}^{+}$ less than $3.0 \mathrm{mmol} / \mathrm{l}$. While the hypokalaemia is often the result of the underlying disease process, $56 \%$ of cases could be considered iatrogenic, due to drug or intravenous fluid administration.

OBERLING, F. (1978). Long-term hypokalaemia in acute myeloid leukaemia. Archives of Internal Medicine, 138, 1287.

MIR, M.A., BRABIN, B., TANG, O.T., LEYLAND, M.J. \& DELAMORE, I.W. (1975). Hypokalaemia in acute myeloid leukaemia. Annals of Internal Medicine, 82, 54

MORGAN, D.P. \& DAVIDSON, C. (1980)..Hypokalaemia and diuretics: an analysis of publications. British Medical Journal, 1, 905.

NABARRO, J.D.N., SPENCER, A.G. \& STOWERS, J.M. (1952). Metabolic studies in severe diabetic ketosis. Quarterly Journal of Medicine, 21, 225.

PICKERING, T.G. \& CATOVSKY, Y.D. (1973). Hypokalaemia and raised lysosyme levels in acute myeloid leukaemia. Quarterly Journal of Medicine, 42, 677.

SCHREINER, G.E. \& MAHER, J.F. (1965). Toxic nephropathy. American Journal of Medicine, 38, 409.

TATTERSALL, M.H., BATTERSBY, G. \& SPIERS, A.S. (1972). Antibiotics and hypokalaemia. Lancet, i, 630.

YOUNG, G.P., SULLIVAN, J. \& HURLEY, T. (1973). Hypokalaemia due to gentamicin/cephalexin in leukaemia. Lancet, ii, 855 . 